\title{
The size variation and related implications of mandibles in northern China in the past 7000 years
}

\author{
LI HaiJun ${ }^{1,2 *}$, ZHANG QuanChao ${ }^{3} \&$ ZHU Hong ${ }^{3}$ \\ ${ }^{1}$ School of Ethnology and Sociology, Minzu University of China, Beijing 100081, China; \\ ${ }^{2}$ Key Laboratory of Evolutionary Systematics of Vertebrates, Chinese Academy of Sciences, Beijing 100044, China; \\ ${ }^{3}$ Research Center for Chinese Frontier Archaeology, Jilin University, Changchun 130012, China
}

Received June 29, 2011; accepted September 13, 2011; published online October 17, 2011

\begin{abstract}
To better understand variation of Holocene Chinese mandiblular morphology, a study was conducted on 23 metric traits of Neolithic $(n=54)$, Bronze-Iron Ages $(n=184)$ and modern $(n=92)$ adult male mandibles from northern China. Results indicate that the linear characters of these Chinese mandibles evolved in the past 7000 years. From the Neolithic to Bronze-Iron Ages to present day, the overall size of mandibles decreased. The linear characters of the mandiblular features varied between different time periods. The decrease of thickness and height of the mandibular corpus primarily occurred during the Neolithic to Bronze-Iron Ages. The decrease in main size was during the Bronze-Iron Ages to present day. It is possible that mandibles became thinner before the overall size decreased. Comparisons also indicate that the bottom part of the face may have decreased more greatly in breadth than the upper portion. The decrease in mandible size may be associated with changes in climate and diet, and with changes in the craniums size.
\end{abstract}

Holocene, Chinese mandibles, morphology microevolution

Citation: $\quad$ Li H J, Zhang Q C, Zhu H. The size variation and related implications of mandibles in northern China in the past 7000 years. Chin Sci Bull, 2012, 57: 387-394, doi: 10.1007/s11434-011-4808-1

Mandibles play an important role in chewing. Studies have shown that mandible morphology is related to food, mastication stress, cranium morphology, population history, middle cranial fossa size and weather [1-5]. Mandible morphology therefore may provide historical information about human food structure, evolution, migration and population relationships [6-8].

Research has shown that human mandibles experienced morphological changes during the Holocene, and European mandibles have decreased in size in recent times [9-11]. A subrecent European population emerged as more different from a recent European population than other more diverse modern populations were from each other, suggesting big morphological plasticity in the mandible through time [12]. Moore and colleagues [11] found that English mandibles decreased from the Neolithic to 20th century, especially in

*Corresponding author (email: Lindavy@163.com) the ramus. Similar change was found by Lavelle [13], who showed that between Romano-British and 19th century periods, English mandibles experienced a reduction in many dimensions, without a corresponding change in teeth. However, it was not clear whether such changes took place mainly in the ramus or corpus part. Another study showed that the overall breadth of Japanese mandibles showed clear narrowing, and remarkable size reduction mainly occurred in the regions of the major masticatory muscle attachments, including coronoid process and the gonial angle [14]. The lateral corpus thickness of the Japanese mandibles also exhibited reduction, which may be the consequence of mandibular size decrease [15]. Other studies have documented similar temporal changes in Japanese mandibles [16,17].

Chinese skulls also have evolved throughout the Holocene. From the Neolithic to present day, general trends include: decrease in cranial and facial dimensions; narrowing and lengthening of the nose; narrowing and heightening of 
the orbits; trend toward a more globular morphology of the head [18]. Thus far, there have been few studies on whether the mandibles of Chinese populations evolved during the Holocene. This paper presents a discussion on morphological change and related implications of Chinese Holocene mandibles, based on linear measurements and comparisons among mandibles from different periods in the Holocene.

\section{Materials and methods}

\subsection{Materials}

To reduce the influence of age, sex and geographic region, we chose 330 adult male mandibles from northern China. These mandibles are at the Institute of Vertebrate Paleontology and Paleoanthropology, Chinese Academy of Sciences, and the Research Center for Chinese Frontier Archaeology of Jilin University. All the mandibles were measured by the first author of this paper.

Miaozigou site. This site is located on the southern hillside of Miaozigou village, Uraharura township, Inner Mongolia, and the age of this site is $5500-5000$ a BP $[19,20]$. Many tools of agricultural production were unearthed. Archaeology and study of stable isotopic ratios of carbon and nitrogen in bone collagen showed that agriculture played a major part in the life of these people, with a smaller contribution from hunting-gathering and animal breeding [20,21].

Jiangjialing site. This site is located in the eastern part of Nihewan Basin, Hebei Province, with an age of about 6850 a BP [22]. Many earthenwares were unearthed at this site [23]. People mainly ate plant products [24].

Xishan site. This site is located in Zhengzhou City, Henan Province, and is about 5300-4800 a BP [25]. Agriculture played a major part in the life of ancient inhabitants here, with a smaller contribution from hunting, fishing and animal breeding [26]. Plants of C4 (probably rice) may have been the main food [27].

Tuchengzi site. This site is located in Helinge'er County, Inner Mongolia. Tuchengzi site contains human skeleton from different periods, such as Warring States Period, Han Dynasty, Tang Dynasty and Qin Dynasty [28]. Most of the mandibles from this site belong to the Warring States Period. These ancient inhabitants were soldiers, mainly eating plant products [29].

Lamadong site. This site is located in Beipiao City, Liaoning Province. This site belongs to the Sanyan Culture, with an age of about 1600-1700 a BP [30]. The values of $\delta^{13} \mathrm{C}$ and $\delta^{15} \mathrm{~N}$ of the collagen showed that these ancestors mainly relied on $\mathrm{C} 4$ plants, and were mainly vegetarians. It is possible that the lifestyle of the Lamadong people (Xianbei people) was strongly influenced by the Han culture, because they were changing from a nomadic phase to a settled farming phase [31].

Guanmashan site. This site is located in Jiutai City, Jilin
Province, and it belongs to the Warring States Period. Earthenware, copperware, stone tools and bone tools were unearthed [32].

Youyao site. This site is located in Xinzhou City, Shanxi Province. Unearthed artifacts included housesites, earthenware, daily-life utensils and tools of production, including copperware. This site contains artifacts belonging to the Xia Dynasty, with a theradiocarbon age of about 4000 a BP [33]. Agriculture played a major role in the life of ancient people here [34].

Datong site. This site is located in Datong City, Shanxi Province, and it belongs to the time of the Northern Wei Dynasty [35].

Zhaimao site. This site is located in Shenmu County, Shaanxi Province, and this site is about $4800-4100$ a BP [36].

Wayaogou site. This site is located in Tongchuan City, Shaanxi Province. This site belongs to the Bronze Age [37].

Longxian site. This site is located in Longxian County, Shaanxi Province, and it contains tombs of the Warring States Period, Han Dynasty and Tang Dynasty [38].

Donghuishan site. This site is located in Minle County, Gansu Province, and it belongs to the Siba Culture, with an age of about 3490 a BP, based on ${ }^{14} \mathrm{C}$ dating [39].

Xiaohandi and Mapai site. This site is located in Minhe County, Qinghai Province, and belongs to the Bronze Age.

North China site. Modern mandibles from North China.

\subsection{Methods}

Some of the sites studied did not contain many mandibles (i.e. Miaozigou, Donghuishan, Guanmashan); Some had unclear ages, and some contained mandibles of different age (i.e. Longxian, Tuchengzi). Mandibles were divided into 3 groups in this paper: Neolithic, Bronze-Iron, and present (Table 1).

We chose 23 mandibular metric traits for analysis (Table 2). Independence-Samples- $T$-tests were used to test for significant differences between time periods for each measurement, using SPSS. Percents of the temporal mandibular

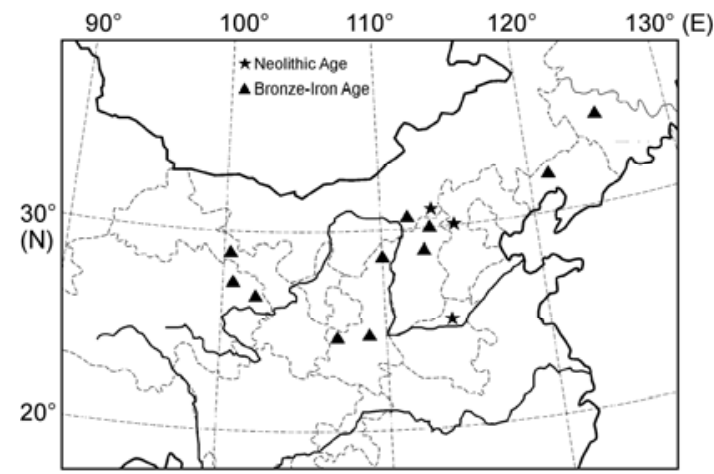

Figure 1 The distribution of the mandibles used in this study (modern mandibles not included). 
Table 1 Mandibles used in this study

\begin{tabular}{ccrl}
\hline Age & Population & $n$ & \multicolumn{1}{c}{ Geographic origin } \\
\hline \multirow{2}{*}{ Neolithic Age (7000-4800 a BP) } & Hebei & 42 & Jiangiialiang \\
& Henan & 8 & Xishan \\
& Inner Mongolia & 4 & Miaozigou \\
\hline & Liaoning & 46 & Lamadong \\
& Inner Mongolia & 88 & Tuchengzi \\
Bronze-Iron Ages (4800-1600 a BP) & Qinghai & 8 & Xiaohandi, Mapai \\
& Gansu & 4 & Donghuishan \\
& Shannxi & 23 & Zhaimao (6) Longxian (13) Wayaogou (4) \\
& Shanxi & 14 & Youyao (7) Datong (7) \\
\hline Present day, modern (100 a BP) & Jilin & 1 & Jiutaishan \\
\hline
\end{tabular}

Table 2 Comparison of the mandible dimension in different periods in Holocene Chinese populations (adult male) ${ }^{\mathrm{a}}$

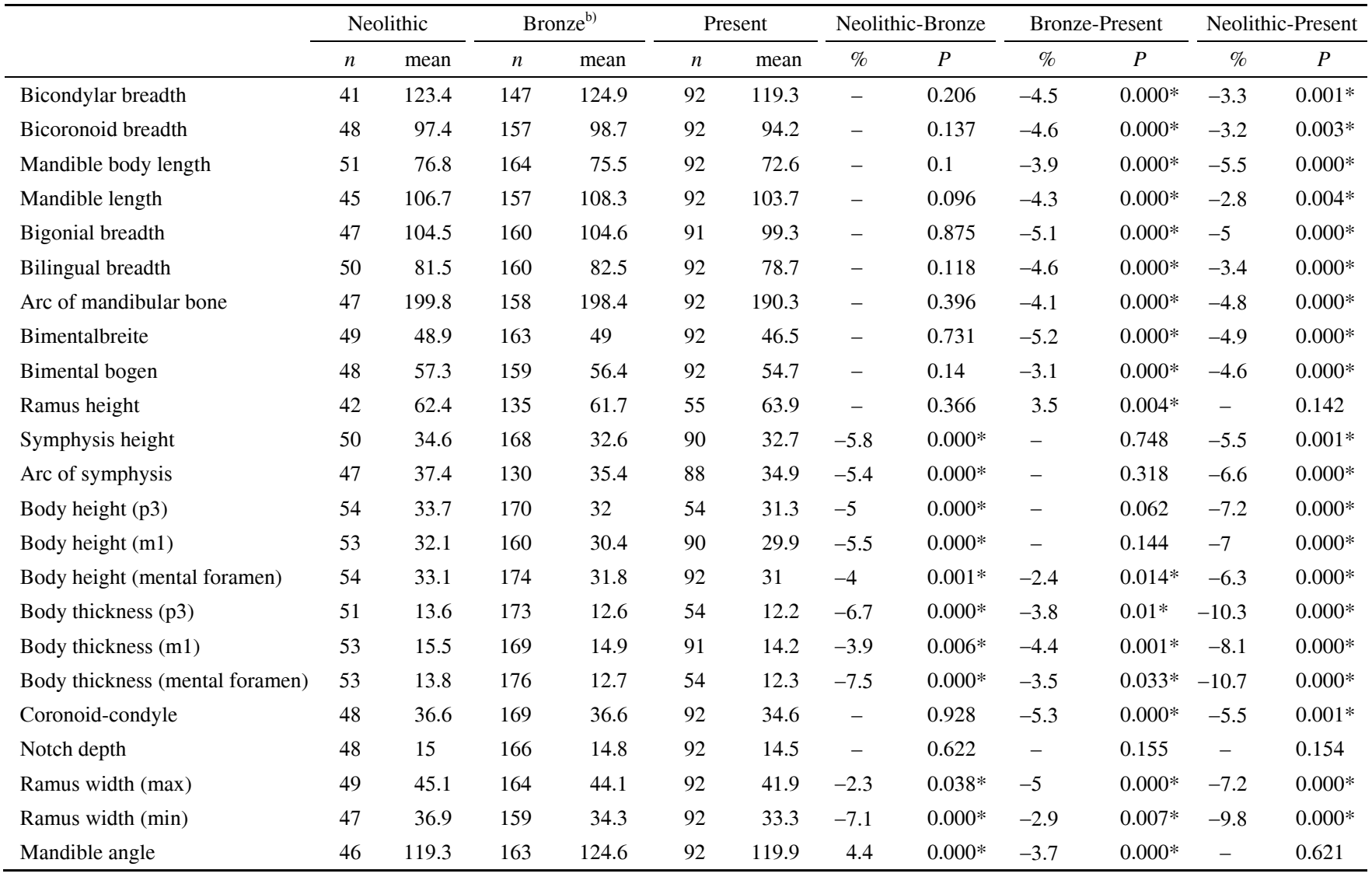

a) Mandible angle $\left({ }^{\circ}\right), \mathrm{mm} ; \alpha=0.05 / 2$, * means $P \leqslant 0.05$, "- " indicates no statistical significance (percents were not computed). b) Bronze stands for "Bronze-Iron" in Tables 2 and 3. Bimentalbreite indicates bimental foramen breadth; bimental bogen indicates arc of mental foramen-mental foramen.

changes were calculated for those traits with significant change $(\alpha=0.05)$, using a formula similar to that of refs. $[18,40]$. The formula is

Temporal mandibular changes $(\%)=100 \times(X 2-X 1) / X 1$, where $X 1$ and $X 2$ stands for average of the same dimension of different cultural periods. The period $X 1$ is older than $X 2$.

When computing the area of mandibular ramus, we re- garded it as rectangular with a triangular hole (Figure 2). Area of mandible ramus $=$ ramus height $\times$ average breadth of mandible ramus - area of notch. The cross section of mandibular corpus was regarded as a rectangular, area of the cross section $=$ body height $\times$ body thickness. Mandibular corpus was regarded as a cuboid, volume = body height $x$ body thickness $x$ arc of mandibular bone. Using this method, there were some common areas between mandible 


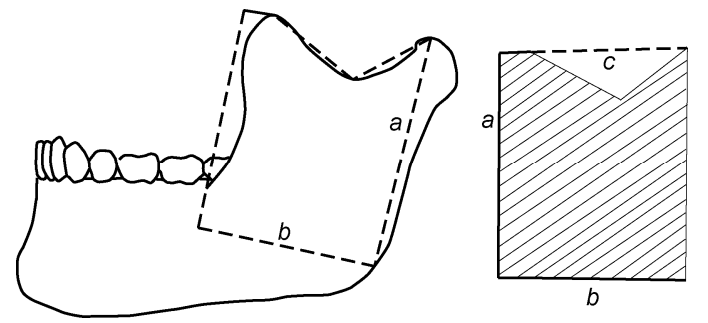

Figure 2 Method to measure the area of mandibular rumas (revised on [14]). $a$, Ramus height; $b$, average ramus width; $c$, coronoid-condyle.

ramus and mandibular corpus. There were no distinct borderlines between these two parts, and the common area was not large. Thus, we found this method to be acceptable. Figure 2 describes the methodology we used to obtain the area of the mandible ramus. In this method, the ramus was a rectangular shape with a triangular hole.

\section{Results}

\subsection{Morphological variations in the mandible between the Neolithic and Bronze-Iron Ages human populations}

From the Neolithic to Bronze-Iron Ages, some traits decreased significantly, including symphysis height, arc of symphysis, body height (p3), body height (m1), body height (mental foramen), body thickness (p3), body thickness (m1), body thickness (mental foramen), ramus width (max), ramus width ( $\mathrm{min}$ ), and mandible angle. Among these traits, symphysis height (decrease by $5.8 \%$ ), body thickness (p3) (decrease by 6.7\%), body thickness (mental foramen) (decrease by $7.5 \%$ ) and ramus width (min) (decrease by $7.1 \%$ ) show a relatively large percent change.

Those traits which have no statistically significant differences included bicondylar breadth, bicoronoid breadth, mandible body breadth, mandible length, bigonial breadth, bilingual breadth, arc of mandibular bone, bimentalbreite, bimental bogen, ramus height, coronoid-condyle and notch depth.

\subsection{Morphological variations in the mandible between Bronze-Iron Ages and modern human populations}

From the Bronze-Iron Ages to present day, some traits had statistically significant differences (ramus height increased, others decreased), including bicondylar breadth, bicoronoid breadth, mandible body breadth, mandible length, bigonial breadth, bilingual breadth, arc of mandibular bone, bimentalbreite, bimental bogen, ramus height, body height (mental foramen), body thickness (p3), body thickness (m1), body thickness (mental foramen), coronoid-condyle, ramus width $(\max )$, ramus width $(\min )$ and mandible angle. All these values were under $5.4 \%$.

From the Bronze-Iron Ages to present day, five traits had no statistically significant differences, including symphysis height, arc of symphysis, body height (p3), body height (m1) and notch depth.

\subsection{Morphological variations in the mandible between Neolithic and present populations}

From the Neolithic to present day, some traits had statistically significant differences, including bicondylar breadth, bicoronoid breadth, mandible body breadth, mandible length, bigonial breadth, bilingual breadth, arc of mandibular bone, bimentalbreite, bimental bogen, symphysis height, arc of symphysis, body height (p3), body height (m1), body height (mental foramen), body thickness (p3), body thickness (m1), body thickness (mental foramen), coronoidcondyle, ramus width (max) and ramus width (min).

Three traits had no statistically significant differences, including ramus height, notch depth and mandible angle.

\subsection{Variations of the ramus during the Holocene}

The percent change of ramus width (max), ramus width (min), coronoid-condyle and notch depth do partly reflect the change of mandible ramus from different angles. In this paper, we chose the measurement of the ramus area to analyze variation of the ramus. We regarded the ramus as a rectangular shape with a triangular hole, and took the average of the ramus width (max) and ramus width ( $\min )$ as the ramus width. The areas and percent change of mandible ramus are given in Table 3 .

Mandible ramus area $=$ ramus height $\times$ ramus width area of mandible notch $=$ ramus height $\times($ ramus width $(\max )$ + ramus width $(\min )) \times 0.5-$ coronoid-condyle $\times$ notch depth $\times 0.5$.

Table 3 Comparison of some areas and volume of mandible of different periods in the Holocene Chinese populations (adult male) ${ }^{\text {a) }}$

\begin{tabular}{|c|c|c|c|c|c|c|}
\hline & \multirow{2}{*}{ Neolithic } & \multirow{2}{*}{ Bronze } & \multirow{2}{*}{ Present } & \multicolumn{3}{|c|}{ Change percentage $(\%)$} \\
\hline & & & & Neolithic-Bronze & Bronze-Present & Neolithic-Present \\
\hline Mandible ramus area & 2283.9 & 2147.8 & 2151.8 & -6.0 & 0.2 & -5.8 \\
\hline Body cross-section area (p3) & 458.3 & 403.2 & 381.9 & -12 & -5.3 & -16.7 \\
\hline Body cross-section area (m1) & 497.6 & 453.0 & 424.6 & -9 & -6.3 & -14.7 \\
\hline Body cross-section area (mental foramen) & 456.8 & 403.9 & 381.3 & -11.6 & -5.6 & -16.5 \\
\hline
\end{tabular}

a) Area in $\mathrm{mm}^{2}$, volume in $\mathrm{mm}^{3}$. 
The percent change of ramus area in different time periods was $-6.0 \%, 0.2 \%,-5.8 \%$ respectively in the Holocene.

\subsection{Variations of mandible corpus in different periods in the Holocene}

Results are presented in Table 3, and the formula is

Mandible body cross section area $=$ body height $\times$ body thickness.

Mandible body volume $=$ arc of mandibular bone $\times$ body height (average) $\times$ body thickness (average).

Body height $($ average $)=[$ body height $(\mathrm{p} 3)+$ body height (m1) + body height (mental foramen)]/3.

Body thickness (average) $=[$ body thickness $(\mathrm{p} 3)+$ body thickness $(\mathrm{m} 1)+$ body thickness (mental foramen) $] / 3$.

The variations of cross-sectional area and volume of mandible body were chosen to show the variation of mandible corpus during different periods. Comparing to the percent change of ramus area, mandible body cross-sectional area and mandible body volume changed more.

\section{Discussion and conclusions}

\subsection{The variations traits of mandible morphology in Holocene Chinese populations}

From the Neolithic to Bronze-Iron Ages to present day, most dimensions of mandibles decreased (Figure 3). These changes indicate that human mandibles were still evolving, which agrees with many other studies [9-11,14,15,17]. Except for ramus height, the decrease of mandible dimensions with statistical significance occurred during the Bronze-Iron Ages to present day, such as bicondylar breadth, bicoronoid breadth, mandible body breadth, mandible length, bigonial breadth, and arc of mandibular bone. Changes in ramus area primarily took place during the Neolithic to Bronze-Iron Ages. Changes in the corpus, such as body thickness, body height, symphysis height, cross section area and body volume also primarily took place during the Neolithic to Bronze-Iron Ages. The thickness of the mandibular body decreased markedly during the Bronze-Iron Ages to present day, but not as much as in the period from the Neolithic to Bronze-Iron Ages. The above changes may indicate that the
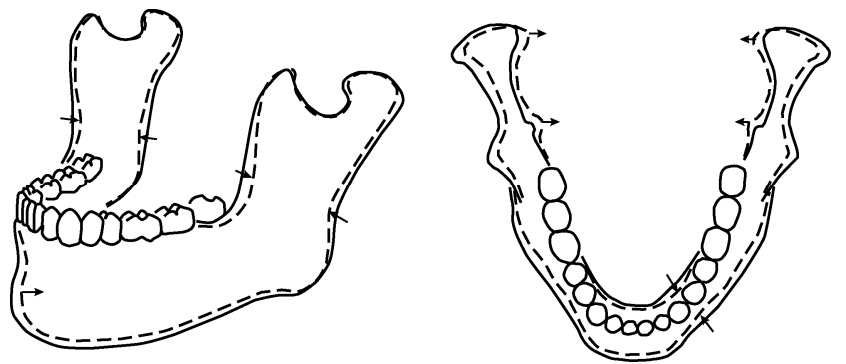

Figure 3 Sketch showing the evolutionary trends of mandible dimensions in Holocene Chinese populations (revised on [14]). changes in mandibular corpus (height and thickness) came before the changes in other dimensions of the mandible. In other words, mandibles became thinner first, and then smaller.

Mandible angle increased during the Neolithic to BronzeIron Ages, but decreased during the Bronze-Iron Ages to present day. This is the only one trait we measured that shows two opposite trends in variation in different time period in the Holocene. The change of mandible angle was closely related to mastication [41]. A smaller angle (near to $90^{\circ}$ ) can help generate greater biting forces than a larger angle [42]. Mandible angle had two different change trends, an increase during the Neolithic to Bronze-Iron Ages, and a decrease during the Bronze-Iron Ages to present day in the Holocene. These trends may reflect the influence of change in food and overall size of the mandible, respectively. $\mathrm{Hu}-$ mans experienced great changes in lifestyle during the Neolithic to Bronze-Iron Ages. The food softening and decrease in masticatory power required may have been the cause of changes in mandible angle, which became greater than a right angle. From Bronze-Iron Ages to present day, human life improved, as did the food and cooking techniques. The mandible size decreased very significantly, which may have made the masticatory power decrease dramatically. The mandible angle needed to become smaller (becoming closer to a right angle) to compensate for the great loss of masticatory power.

The change in mandible angle also may be closely related to mandible body length, mandible length and ramus height. The relationship of mandible body length $(a)$, mandible length $(b)$, ramus height $(d)$, and mandible angle $\left(180^{\circ}-\angle A\right)$ is shown in Figure 4. $\cos \angle A \approx(b-a) / d, b-a=$ $c$. Inserting the average data from Table 2 into the formula above, $\cos \angle A$ (Neolithic) $=0.479, \cos \angle A$ (Bronze-Iron $)=$ $0.532, \cos \angle A$ (Present) $=0.487$. Thus, mandible angle

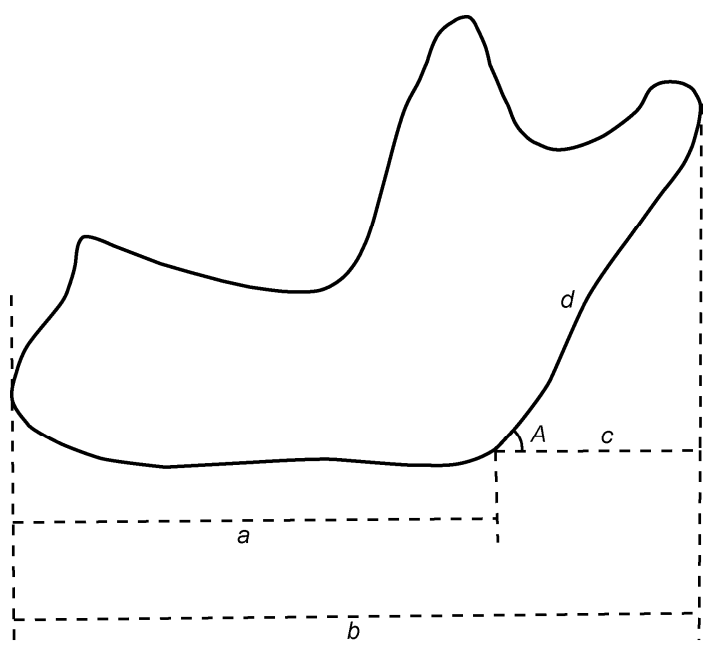

Figure 4 Mandible angle and some related measurements. $a$, Mandible body length; $b$, mandible length; $c=b-a$; $d$, ramus height; $\angle A=180^{\circ}-$ $\angle A ; \cos \angle A \approx c / d=(b-a) / d$. 
$\left(180^{\circ}-\angle A\right)$ : Bronze-Iron $>$ Present $>$ Neolithic, which matches with the mandible angle data relationship very well (Table 2), indicating that the change of mandible angle was closely related to mandible body length, mandible length and ramus height.

Ramus height increased from Bronze-Iron Ages to present day. During the same time period, the overall breadth measurements of mandible showed marked narrowing, upper facial height [18] and symphysis height increased (although not statistically significant), and bizygomatic breadth decreased [18]. The change in ramus height may indicate that the face became narrow and tall. A previous study measured masticator power differences between people with shorter vs. taller faces, and found that shorter-faced human group had bigger masticatory power than taller-faced group [42]. The change in face height also may have been caused by a decrease in masticatory power, which is related to diet softening.

The change of mandible ramus area mainly occurred during the Neolithic to Bronze-Iron Ages. Mandible ramus assissted in chewing [13], therefore its size change may indicate that a decrease in masticatory power mainly took place during the Neolithic to Bronze-Iron Ages.

Research on teeth malocclusion also supports the view that mandibles decreased in the Holocene. The percent occurrence of teeth malocclusion of the Baoji-Huaxian Group in the Neolithic was 26.3\% [43]. In the Bronze-Iron Ages, the percent of the Anyang group and Lamadong group were $28 \%$ [44] and $42.53 \%$ [45], respectively. The percent in modern people is greater. Chen and colleagues [46] reported a frequency of $69.46 \%$, according to an investigation of 1500 university students. Chen and colleagues [47] found that the percent occurrence in modern people is up to $80.15 \%$, which is extremely high. The symptom of malocclusion includes dentition crowding, and discordance of teeth with alveolar bone. Considering the relatively small change in tooth dimensions in the past thousands years $[9-11,48]$, the higher percentage of malocclusion may be caused by a decrease in mandibles.

\subsection{Comparisons between variation of mandibles and craniums}

Comparisons of morphological variations of mandibles and craniums [18] in the Holocene reveals that bizygomatic breadth, bicondylar breadth, bicoronoid breadth experienced similar changes in the Holocene. These three dimensions showed no significant change during the Neolithic to Bronze-Iron Ages, and the percent variation was $-3.2 \%$, $-3.3 \%,-3.2 \%$, respectively, during the Neolithic to present day (Tables 2 and 4). Such similarity may partly be due to their close anatomical positions. Cranial breadth, bizygomatic breadth, bigonial breadth decreased by $2 \%, 3.2 \%$, and $5 \%$, respectively, from the Neolithic to present day (Tables 2 and 4), which may indicate that the narrowing of lower facial parts was more obvious than that of the upper parts.

Cranium changed more obviously during the Bronze-Iron Ages to present day than during the Neolithic to BronzeIron Ages (Table 4). The main change in mandibles also took place during the Bronze-Iron Ages to present day. Such consistency may not be a coincidence, but may be due to internal influences. Mandibles are located in the lower part of the facial area, and are connected to the cranium through temporomandibular joints. Together with the cranium, mandibles form the facial profile and play a role in mastication. Considering anatomical and functional relationships between mandible and cranium, it is likely that these two units evolved together. The morphological change in cranium may have induced morphological changes in the mandible, and vise versa.

However, there are certain limitations in such comparisons. The mandibles and craniums are not regionally and individually compatible. The crania used in these studies were not from the same individuals as the mandibles. We plan to conduct further studies based on mandibles and craniums from the same individuals.

Table 4 Comparison of the cranial percent change of different time periods in Holocene Chinese populations (computed according to [18])

\begin{tabular}{|c|c|c|c|}
\hline & Neolithic-Bronze-Iron Ages & Bronze-Iron Ages-Present day & Neolithic Age-Present day \\
\hline & Change percentage $(\%)$ & Change percentage $(\%)$ & Change percentage $(\%)$ \\
\hline Cranial length & 1.8 & -2.9 & -1.1 \\
\hline Cranial height & -1.8 & -1.5 & -3.3 \\
\hline Basis length & -2.3 & -2.9 & -5.2 \\
\hline Profile length & -3 & -3.2 & -6.1 \\
\hline Upper facial height & -1.8 & 0.4 & -1.4 \\
\hline Bizygomatic breadth & 0 & -3.2 & -3.2 \\
\hline Orbital breadth & -3 & -3.6 & -6.5 \\
\hline Orbital height & 0.6 & 3.2 & 3.8 \\
\hline Nasal breadth & -1.8 & -6 & -7.7 \\
\hline Nasal height & 0.2 & 0.7 & 0.9 \\
\hline
\end{tabular}




\subsection{Possible reasons for the mandible morphological variations in Holocene populations}

Many studies have been conducted on the causes of morphological variations of mandibles in the Holocene. Some researchers believe that the variations are closely related to food structure and mastication functions. The variation of mandible linear dimensions mainly is influenced by ontogenetic and masticatory function [49,50]. The decrease in mandibles probably is caused by the food structure softening, which provided less stimulation for mandibles to grow [51,52]. Saitou [53] simulated the food of different periods and conducted chewing experiments, and found that in recent periods, the masticatory cycles and time were lessening. Kaifu [15] summarized the possible effects of diminishing chewing stress on mandibles as follows: (1) overall narrowing, (2) weakening in the regions of major masticatory muscle attachments, (3) thinning of body thickness, and (4) thinning of cortical bone thickness at symphysis. Other studies have shown that the reduction in mandible dimension is only a part of the entire cranium evolution, and may not have much relationship with food change [54,55]. Kaifu believed that the change in corpus thickness may reveal certain genetic transformations and mixture [15], and the increase in symphysis height in Kanto may be the result of a genetic mixture from immigrants [14]. Nicholson et al. [56] believed that the morphology of the mandible may be affected by many factors, such as climate change, masticatory function, and human gene communication.

Lavelle [13] suggested that if reduction in mandibular dimensions were mainly caused by food changes, then the mandibular ramus would be more reduced than the body. Comparing the percent change of ramus area with mandible body cross-sectional area, as well as mandible body volume, we can see that the mandible body changed more significantly than the ramus, which may indicate that evolution may be the main reason for the morphological variation in mandibles. Moreover, it may be difficult to identify whether the food change or evolution trend is the main reason when physical traits experienced long term and stable variations. This is because food changes also may accelerate the process of evolution.

Agriculture played a major role in the lives of ancient inhabitants in Miaozigou (Miaozigou and Jiangjialiang people share similar cranium and dental traits [57]), Jiangjialiang, Xishan, Tuchengzi, Lamadong and Youyao. In addition, plant products were the main food for these people, $81.9 \%$ of the total mandibles from the archaeological sites (Neolithic and Bronze-Iron Ages). According to geographical position, other ancient inhabitants from Guanmashan, Datong, Zhaimao, Wayaogou, Longxian, Donghuishan, Xiaohandi and Mapai, about $18.1 \%$ of the total, possibly ate mainly plant products. Generally speaking, people from North China also ate mainly plant products. The similar food structures provide a good background for analyzing the reason for mandible variations. The variation of mandibles may have been caused by changes in the way of cooking and the food structure within plant products.

Climate changed greatly during the Holocene. There was a major eruptive environmental deterioration all over the world around 5000 years ago, which was the main cause for the decline in Chinese Neolithic culture [58]. The Yellow River basin suffered a sudden climate change, which caused a drought at around $2000 \mathrm{BC}[59,60]$. This environmental change may have caused dietary changes, and transformed prehistoric cultures [58]. All the factors above may have influenced human's physical traits. Hence, the variation of mandibles during Neolithic to Bronze-Iron Ages from our study sites may be the consequence of the above changes. During the Bronze-Iron Ages, human lifestyles experienced further change with the food resources becoming abundant. With the wide spread of mental tools and technology development in cooking food (food softer and easier to chew), there may have been a subsequent weakening of chewing power and development of a smaller sized mandibles. However, what may have caused the mandible change in this time period is not quite clear, and further work is needed to elucidate this issue.

This work was supported by the National Natural Science Foundation of China (41102015), the Projects from the Chinese Academy of Sciences (XDA05130102 and KZCX2-YW-159), and the Ministry of Science and Technology of China (2009DFB20580).

1 Liu W, Jin C Z, Zhang Y Q, et al. Human remains from Zhirendong, South China, and modern human emergence in East Asia. Proc Natl Acad Sci USA, 2010, 107: 19201-19206

2 Igarashi Y, Ohzeki S, Uesu K, et al. Frequency of mandibular tori in the present-day Japanese. Anthropol Sci, 2008, 116: 17-32

3 Fukase H, Suwa G. Growth-related changes in prehistoric Jomon and modern Japanese mandibles with emphasis on cortical bone distribution. Am J Phys Anthropol, 2008, 136: 441-454

4 Bastir M, Rosas A, Kuroe K. Petrosal orientation and mandibular ramus breadth: Evidence for an integrated petroso-mandibular developmental unit. Am J Phys Anthropol, 2004, 123: 340-350

5 Puişoru M, Forna N, Fătu A M. Analysis of mandibular variability in humans of different geographic areas. Ann Anatomy, 2006, 188: 547-554

6 Mahoney P. Microwear and morphology: Functional relationships between human dental microwear and the mandible. J Hum Evol, 2006, 50: 452-459

7 Shang H, Tong H W, Zhang S Q, et al. An early modern human from Tianyuan Cave, Zhoukoudian, China. Proc Natl Acad Sci USA, 2007, 104: 6573-6578

8 Nicholson E, Harvati K. Quantitative analysis of human mandibular shape using three dimensional geometric morphometrics. Am J Phys Anthropol, 2006, 131: 368-383

9 Lysell L. A biometrical study of occlusion and dental arches in a series of mediaeval skulls from northern Sweden. Acta Odontol Scand, 1958, 16: 177-203

10 Goose D H. Reduction of palate size in modern population. Arch Oral Biol, 1962, 7: 343-350

11 Moore W J, Lavelle C L, Spence T F. Changes in the size and shape of the human mandible in Britain. Br Dent J, 1968, 125: 163-169

12 Humphrey L T, Dean M C, Stringer C B. Morphological variation in great ape and modern human mandibles. J Anat, 1999, 195: 491-513

13 Lavelle C L B. A comparison between the mandibles of Romano- 
British and nineteenth century periods. Am J Phys Anthropol, 1972, 36: $213-220$

14 Kaifu Y. Changes in mandibular morphology from the Jomon to modern periods in Eastern Japan. Am J Phys Anthropol, 1997, 104: 227-243

15 Kaifu Y. Temporal changes in corpus thickness of the Japanese mandibles. Bull Natl Sci Mus Ser D, 2000, 26: 39-44

16 Kaifu Y. Regional variation in mandibular morphology of the Jomon people. Bull Natl Sci Mus, 1995, 21: 37-50

17 Maeda T. Mandibular ramus morphology of the Jomon people from Hokkaido. Anthropol Sci, 2002, 110: 27-40

18 Wu X J, Liu W, Zhang Q C, et al. Craniofacial morphological microevolution of Holocene populations in northern China. Chin Sci Bull, 2007, 52: 1661-1668

19 Wei J. Neolithic site in Miaozigou site, Qahar Youyi Qianqi, Inner Mongolia. Yearbook of Chinese Archaeology (1987) (in Chinese). Beijing: Cultural Relics Press, 1988. 119

20 Wei J. On Miaozigou and Dabagou. In: Inner Mongolia Research Institute of Cultural Relics and Archaeology, ed. Collected Works on Primitive Culture in South Central of Inner Mongolia (in Chinese). Beijing: Ocean Press, 1991. 113-118

21 Zhang Q C, Jacqueline T, Wei J, et al. Paleodietary studies using stable Carbon and Nitrogen Isotopes from human bone: An example from the Miaozigou Site, Qahar Youyi Qianqi, Inner Mongolia (in Chinese). Acta Anthropol Sin, 2010, 29: 270-275

$22 \mathrm{Li} \mathrm{F} \mathrm{J,} \mathrm{Zhu} \mathrm{H.} \mathrm{Observation} \mathrm{and} \mathrm{investigation} \mathrm{of} \mathrm{the} \mathrm{skulls} \mathrm{of} \mathrm{Jiangjia-}$ liang Neolithic age site in Yangyuan, Hebei with special reference to nonmetric cranial traits (in Chinese). Acta Anthropol Sin, 2003, 22: 206-217

23 Hebei Provincial Institute of Cultural Relics. Excavation at the Neolithic Jiangjialiang site in Yangyuan county, Hebei (in Chinese). Archaeol, 2001, 113-127

24 Li F J. A Research on Human Skeletons of the Neolithic Age in Jiangjialiang, Yangyuan, Hebei, China (in Chinese). Beijing: Science Press, 2008

25 The Training Program for Archeological Team Directors Under the State Bureau of Cultural Relics. Excavation of a Yangshao site of city in the outskirts of Zhengzhou (in Chinese). Cult Relics, 1999, 4-15

26 Chen Q J. A study of the faunal remains from the Xishan site in Zhengzhou (in Chinese). Acta Archaeol Sin, 2006, 385-418

27 Zhang X L, Qiu S H, Zhong J, et al. Studies on diet of the ancient people of the Yangshao cultural sites in the central plains (in Chinese). Acta Anthropol Sin, 2010, 29: 197-207

28 Inner Mongolia Research Institute of Cultural Relics and Archaeology. The main gain of the excavation of Tuchengzi site in Helinge'er county (in Chinese). Inner Mongolia Cult Relic Archaeol, 2006, 9-16

29 Gu Y C. A Research on the skeletons of Warring-states period from Tuchengzi site, Helinge'er county, Inner Mongolian (in Chinese). Dissertation for the Doctoral Degree. Changchun: Jilin University, 2007

30 Zhang Q C. Dental disease of the Three-yan cultures from Lamadong cemetery of Beipiao (in Chinese). Acta Anthropol Sin, 2003, 22: 29-36

31 Dong Y, Hu Y W, Zhang Q C, et al. Stable Isotopic analysis on human bones of the Lamadong site, Beipiao, Liaoning Province (in Chinese). Acta Anthropol Sin, 2007, 26: 77-84

32 Jilin Provincial Institute of Cultural Relics and Archaeology. Cemetery of Xituanshan culture at Shilashan and Guanmashan site in Jiutai City, Jilin Province (in Chinese). Archaeology, 1991, 337-345

33 Xinzhou Archaeological Team. The excavation of Youyao site in Xinzhou of Shanxi Province (in Chinese). Archaeology, 1989, 289-299

34 Xinzhou Archaeological Team. Archaeology Excavation at Youyao Site, Xinzhou City (in Chinese). Beijing: Science Press, 2004. 1-220

35 Zhang Z B. Morphological variation of dentition in two ancient inhabitants, China (in Chinese). Acta Anthropol Sin, 1993, 12: 103-112

36 Shaanxi Provincial Institute of Archaeology. The excavation of Zhaimao site in Shenmu County (in Chinese). Archaeol Cult Relics,
2002, 3-18

37 Chen L. Cranial features of Bronze Age human remains from Wayaogou, Shaanxi Province (in Chinese). Acta Anthropol Sin, 2000, 19: $32-43$

38 Liu W, Zeng X L. The dental morphology of the people of Zhanguo period in Longxian, Shaanxi Province (in Chinese). Acta Anthropol Sin, 1996, 15: 302-314

39 Gansu Provincial Institute of Cultural Relics and Archaeology, Research Office of Archaelogy of the North at Jilin University. Excavation Report of the Donghuishan Site in Minle-excavation and Study on the Cemetery of Siba Culture (in Chinese). Beijing: Science Press, 1998

40 Zhang Z B. Prove of the origin of modern Chinese spatiotemporal variation of cranium traits (in Chinese). Quat Sci, 1999, 19: 113-124

41 Bai H Y. Age changes of the mandibular angel in Chinese (in Chinese). Acta Anatom Sin, 1979, 10: 13-20

42 Hunt N. Muscle function and the control of facial form. In: Harris M, Edgar M, Meghji S, eds. Clinical Oral Science. Oxford: Wright, 1998. $120-133$

43 Zeng X L, Huang J F, Lin J X. Malocclusion of Neolithic human skeletons from Baoji and Huaxian (in Chinese). Acta Anthropol Sin, 1983, 2: 352-358

44 Mao H, Yen Y. Dental condition of the Shang Dynasty skulls excavated from Anyang and Huixian (in Chinese). Paleovert Paleoanthropol, 1959, 1: 81-85

45 Zhang Q C, Liu Z, Zhu H. The malocclusion of the skeleton of the Wei-Jin Period from Lamadong cemetery of Beipiao (in Chinese). Acta Anthropol Sin, 2003, 22: 321-327

46 Chen Y G, Xing M, Yu L, et al. Investigation and analysis of malocclusion in undergraduate students in Xuzhou (in Chinese). Chin J Aesthetic Med, 2008, 17: 1057-1059

47 Chen Y P, Dong Y J, Sun X H, et al. Investigation and analysis of malocclusion in early stage of permanent teeth (in Chinese). J Oral Sci Res, 2000, 16: 107-109

48 Kaifu Y. Changes in alveolar prognathism and anterior teeth protrusion in Japan. Anthropol Sci, 1999, 107: 3-24

49 Hrdlička A. Mandibular and maxillary hyperostosis. Am J Phys Anthropol, 1940, 27: 1-55

50 Hrdlička A. Lower jaw. Am J Phys Anthropol, 1940, 27: 281-308

51 Wallace J S. Variation in the form of the jaws. Baillière, Tindall and Cox, London. 1927

52 Watt D G, Williams C H M. The effects of the physical consistency of the food on the growth and development of the mandible and maxilla of the rat. Am J Orthodont, 1951, 37: 895-928

53 Saitou S. Mastication and mechanocitology. In: Introduction to the Masticatory System. Tokyo: Fujinsha, 1987. 115-129

54 Keith A. Concerning certain structural changes which are taking place in our jaws and teeth. Dental Board of the United Kingdom, London, 1924

55 Hooton E A. The evolution and devolution of the human face. Am J Orthodont, 1946, 32: 657-672

56 Nicholson E, Harvati K. Quantitative analysis of human mandibular shape using three-dimensional geometric morphometrics. Am J Phys Anthropol, 2006, 131: 368-383

57 Li F J, Zhu H. A dental morphological observation on the Neolithic human skeletons from Jiangjialiang, Yangyuan, Hebei Province (in Chinese). Acta Anthropol Sin, 2006, 25: 87-101

58 Zhu Y, Chen F H, Zhang J W, et al. A discussion on the effects of deteriorated environment event on the Neolithic culture of China, around 5000 a BP (in Chinese). Prog Geogr, 2001, 20: 111-121

59 Zhang L S, Fang X Q, Ren G Y, et al. Environmental changes in the north China farming-grazing transitional zone (in Chinese). Front Earth Sci, 1997, 1-2: 127-136

60 Zhu K Z. Primary study of climatic changes during recent 5000 years in China (in Chinese). Sci China, 1973, 16: 168-189

Open Access This article is distributed under the terms of the Creative Commons Attribution License which permits any use, distribution, and reproduction in any medium, provided the original author(s) and source are credited. 